\title{
Ultrasonic Assisted Synthesis of Cationic Polyacrylamide and Its Dewatering performance evaluation
}

\author{
Xiang $\mathrm{Li}^{1^{*} \text {, a }}$, Baojun Yang ${ }^{2, \mathrm{~b}}$, Qianying Shou ${ }^{3, \mathrm{c}}$, Guoming Zeng ${ }^{1, \mathrm{~d}}$, Pei Wu ${ }^{1, \mathrm{e}}$ \\ ${ }^{1}$ School of Civil Engineering and Architecture, Chongqing University of Science and Technology, \\ Chongqing 401331, China \\ ${ }^{2}$ Key Laboratory of Biorheological Science and Technology (Chongqing University), Ministry of \\ Education, Chongqing 400030, China \\ ${ }^{3}$ Architectural design \& research institute Co., Ltd., Chongqing 400044, China

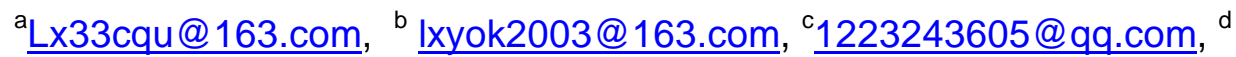 \\ zeng373064894@126.com ${ }^{\mathrm{e}}$ 555wp555@163.com
}

\begin{abstract}
Keywords: Water treatment chemicals, Flocculants, Cationic polyacrylamide, Dyeing sludge, Dewatering

Abstract: In this study, an acid and alkali resistance cationic polyacrylamide flocculants (P(AM-MAPTAC)) was synthesized through copolymerization of acrylamide (AM) and methacrylamido propyl trimethyl ammonium chloride (MAPTAC) by innovative ultrasonic initiation technology. The structure and thermal decomposition property of the obtained polymer were observed through FTIR and TG/DSC measurements respectively. The dewatering performance of P(AM-MAPTAC) was also evaluated by measuring the supernatant residual turbidity (RT), moisture content of the filter cake (FCMC), and chemical oxygen demand (COD) removal rate of the sludge. The RT, FCMC, and COD reached $14.80 \mathrm{NTU}, 83.72 \%$, and 66.8\%, respectively, at $40 \mathrm{mg}$ $\mathrm{L}^{-1}$ of $\mathrm{P}(\mathrm{AM}-\mathrm{MAPTAC})$ and $\mathrm{pH}$ of 10.0. Furthermore, $\mathrm{P}$ (AM-MAPTAC) showed good acid and alkali resistance, and had better application value.
\end{abstract}

\section{Introduction}

With the rapid development of printing - dyeing industry, the amount of wastewater treatment is increasing [1, 2]. Gradually, excess dyeing sludge production increased. As a by-product of wastewater treatment, dyeing sludge which usually features high water content, complex composition and high content of heavy metals has been considered to be a kind of solid waste with high biological toxicity[3, 4]. In addition, the sludge is a kind of hydrophilic biological particles with colloidal network structure. And thus, the formed stable bacterial micelles seriously hinder the dewatering of sludge[5, 6]. Therefore, improving the sludge dewatering performance has become the key of dyeing sludge reduction.

Cationic polyacrylamide (CPAM) is a kind of organic polymer with high positive charge density and relatively high molecular weight. The formed positively charged polymer electrolyte after dissolved in water can give full play to its electrical neutralization and adsorption bridging effect, so as to effectively flocculate compounds and colloidal substances in wastewater, so that these substances are easier to separate from water[7, 8]. Because CPAM has the multiple function of turbidity removal, decolorization, adsorption, filtration and purification, it has been widely used in environment field, especially in sludge dewatering $[9,10]$. Cationic polyacrylamide has shown excellent dewatering performance in sewage/dyeing sludge dewatering in the preliminary study of our research group[11-13]. With the development of printing - dyeing industry and sewage treatment facilities, the amount of sludge emissions increased substantially. This is bound to produce a greater demand for the types and dosages of flocculants. CPAM as an economic, safe and environmental friendly flocculants will also be more widely used.

CPAM usually synthesized throng the method of polyacrylamide cationic modification and copolymerization of acrylamide monomers with cationic monomers. Compared with cationic 
modification, copolymerization method has been widely studied and applied[5, 12]. Because CPAM with high molecular weight and cation degree can be obtained through this method. CPAM obtained through traditional thermally initiated aqueous solution copolymerization feature good product performance. However, disadvantages of high energy consumption caused by long time heating and high residue of neurotoxic monomer caused by low conversion in this synthesis method promote researchers to improve or develop new synthesis method to overcome the above shortcomings. As a feasible, efficient and easy controlled initiation method, ultrasound has been used in the copolymerization of organic polymer flocculants. Ma et al. introduced ultrasound initiation into grafting modification of Chitosan and synthesized cationic chitosan flocculants which showed excellent flocculation performance[14].

In view of all the aforementioned aspects, this work aim at developing environmentally friendly, efficient and energy efficient new synthesis technology for cationic polyacrylamide P(AM-MAPTAC). Furthermore, the sludge dewatering performance of the obtained cationic polyacrylamide taking dyeing sludge as the treatment object, RT, FCMC and COD removal rate as the evaluating indicator was also evaluated in detail.

\section{Experimental}

\section{Materials}

The following materials were used in this work: AM, commercial PAM and P(AM-DAC) (industrial grade; Chongqing Lanjie Tap Water Company, Chongqing, China), MAPTAC (50 wt\% in water and industrial grade; Nanjing Jingruijiuan Biotechnology Co., Ltd. Nanjing, China), and VA-044 (analytical reagent grade; Ruihong Biological Technology, Shanghai, China). Absolute ethyl alcohol, and acetone (analytical reagent grade) were purchased from Chongqing Chuandong Chemical Industry Co., Ltd (Chongqing China). All reagents were used in the experiment without further purification. Raw dyeing sludge samples were collected from the sludge thickener of a printing and dyeing works (Chongqing, China).

\section{Ultrasonic Assisted Synthesis of P(AM-MAPTAC)}

According to the experimental design, a certain proportion of AM and MAPTAC were added into a $10 \mathrm{~mL}$ glass reactor which contains moderate distilled water and dissolved completely. The $\mathrm{pH}$ of the reaction solution was adjusted by adding sodium hydroxide $\left(0.1 \mathrm{~mol} \mathrm{~L}^{-1}\right)$ or hydrochloric acid $(0.1 \mathrm{~mol}$ $\left.\mathrm{L}^{-1}\right)$. Then the glass reactor was bubbled with pure $\mathrm{N}_{2}(99.99 \%)$ for $30 \mathrm{~min}$ to absolutely remove oxygen before adding the photo initiator VA-044. Finally, the glass reactor was placed in an ultrasonic reactor until the set time. After the ultrasonic initiation polymerization, the reaction mixture was allowed to stand and cure for $2 \mathrm{~h}$. The copolymerization polymers were then purified with acetone and ethanol and dried in a vacuum oven at $60{ }^{\circ} \mathrm{C}$.

\section{Characterization of copolymers}

After copolymers were milled into fine powder, FTIR spectra of copolymer were recorded by a 550Series II infrared spectrometer (BRUKER Company, Switzerland) using KBr pellets. The thermal decomposition property of copolymer was determined by a STA449C instrument (Netzsch, Germany) under argon atmosphere at a heating rate of $10^{\circ} \mathrm{C} \mathrm{min}^{-1}$.

\section{Dewatering performance evaluation}

All the P(AM-MAPTAC) flocculants used in dewatering tests were synthesized in laboratory except the commercial $\mathrm{P}(\mathrm{AM}-\mathrm{DAC})$. The dyeing sludge sample presented an initial $\mathrm{pH}$ of 10.0 and $98.8 \%$ moisture content. Dewatering tests were carried out on a program-controlled jar-test apparatus (ZR4-6, Zhongrun Water Industry Technology Development Co., China) at room temperature. The dewatering performance of $\mathrm{P}(\mathrm{AM}-\mathrm{MAPTAC})$ was investigated using residual turbidity (RT) of supernatant after flocculation as well as filter cake moisture content (FCMC) and specific resistance to filtration (SRF) of sludge as evaluation indicators. $1 \mathrm{~g} \mathrm{~L}^{-1}$ of flocculants were pre prepared by dissolve a certain amount of solid flocculants in $100 \mathrm{~mL}$ distilled water. In the test, set dosage of flocculants was 
added into $250 \mathrm{ml}$ beaker with $100 \mathrm{~mL}$ initial dyeing sludge. Then the $\mathrm{pH}$ of the solution was adjusted by adding sodium hydroxide $\left(0.1 \mathrm{~mol} \mathrm{~L}^{-1}\right)$ and hydrochloric acid $\left(0.1 \mathrm{~mol} \mathrm{~L}^{-1}\right)$. The solution was rapidly stirred at $120 \mathrm{rpm}$ for $20 \mathrm{~s}$, followed by $40 \mathrm{rpm}$ for $60 \mathrm{~s}$ and then stood for $10 \mathrm{~min}$. After flocculation and settling process, the supernatant $1 \mathrm{~cm}$ below the water surface was collected for RT, COD and Zeta potential measure, and then the conditioned sludge samples were filtered through a vacuum suction filter machine under a pressure of $0.05 \mathrm{MPa}$ for $30 \mathrm{~min}$ for the FCMC and SRF measure.

\section{Results and discussion}

\section{Structure identification}

FTIR spectra of P(AM-MAPTAC): Fig. 1(a) shows the FTIR spectra of AM. As shown in Fig. 1(a), the peaks at $1530,1491,1450$ and $970 \mathrm{~cm}^{-1}$ which represent the deformation vibration of methylene, quaternary ammonium methyl and mono substituted methyl ammonium $(\mathrm{N}-\mathrm{H})$, stretching vibration of $-\mathrm{N}^{+}\left(\mathrm{CH}_{3}\right)_{3}$ in MAPTAC unit were observed[15]. The peaks at 3438 and $1668 \mathrm{~cm}^{-1}$ which represent $-\mathrm{NH}_{2}$ and $\mathrm{C}=\mathrm{O}$ in $\mathrm{AM}$ unit were also observed[16, 17]. Therefore, all these characteristic peaks confirmed that the two monomers were successfully copolymerized.

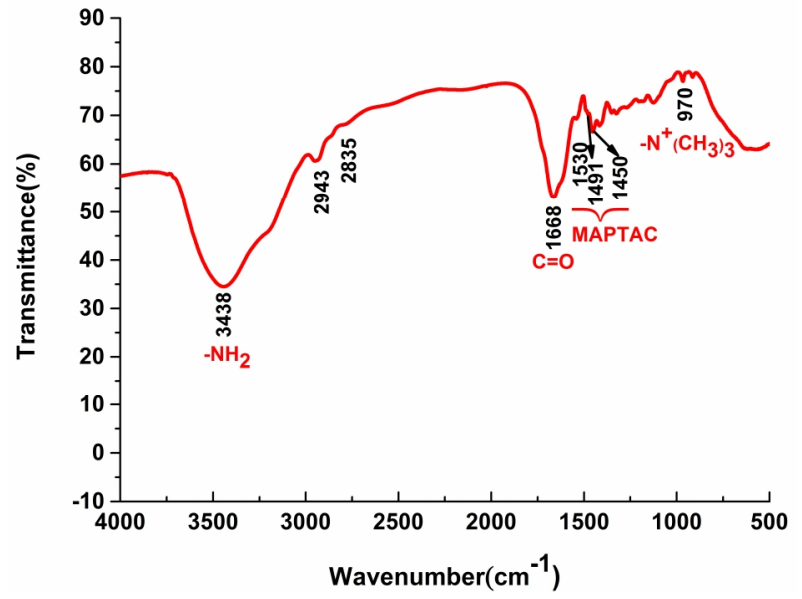

a

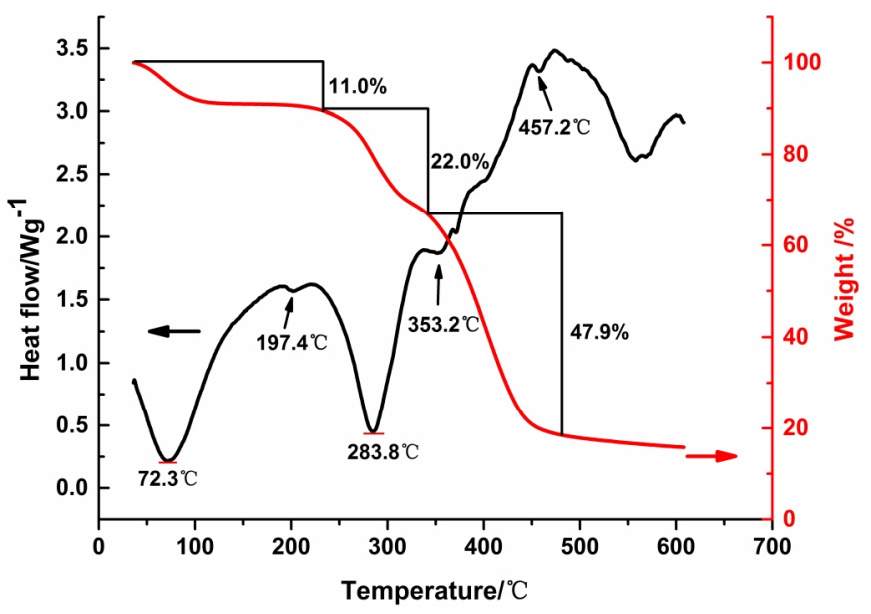

b

Figure 1 FTIR spectra and TG/DSC analysis of P(AM-MAPTAC)

TG/DSC analysis of P(AM-MAPTAC): Fig. 1(b) shows the TG/DSC analysis of P(AM-MAPTAC). As shown in Fig. 1(b), three obvious weight loss stages on the thermal weight curve of $\mathrm{P}(\mathrm{AM}-\mathrm{MAPTAC})$ were observed. The first stage $\left(35.2 \sim 196.8^{\circ} \mathrm{C}, 11.0 \%\right.$ of weight loss) was the moisture loss; The second stage $\left(196.8 \sim 352.0^{\circ} \mathrm{C}, 22.0 \%\right.$ of weight loss) was considered for the thermal decomposition and imidization of amide groups (-CO-NH-), detachment of methyl from quaternary ammonium $\left(-\mathrm{C}\left(\mathrm{CH}_{3}\right)_{3} \mathrm{~N}^{+} \mathrm{Cl}^{-}\right)$and the removal of hydrogen chloride $[17,18]$. The third stage (352.0 497. $2^{\circ} \mathrm{C}, 47.9 \%$ of weight loss) was the decomposition of polymer backbone.

\section{Dewatering performance evaluation}

As a traditional industry, dyeing and printing industry plays an important role in the national economy. However, a large number of printing and dyeing wastewater and sludge have been restricting the prosperity and development of this traditional industry. High water content is a major characteristic of sludge refractory. Therefore, developing reasonable and effective methods to reduce the moisture content of dyeing sludge has become the key of dyeing sludge treatment. In this section, the dewatering performance of $\mathrm{P}$ (AM-MAPTAC) (14.3 $\mathrm{dL} \cdot \mathrm{g}^{-1}$ of intrinsic viscosity, $25 \%$ of cationic degree) on dyeing sludge was investigated in detail taking RT, FCMC, SRF and COD as the evaluating indicator. 
Furthermore, the contrast treatment effect between P(AM-MAPTAC) and commercial P(AM-DAC) $\left(14.5 \mathrm{dL} \cdot \mathrm{g}^{-1}\right.$ of intrinsic viscosity, $25 \%$ of cationic degree) were also studied.

\section{Effect of dosage on dewatering performance}

The effect of dosage on RT, FCMC, SRF and COD are shown in Fig. 2. As shown in Fig. 2, When the dosage of P (AM-MAPTAC) was less than $40 \mathrm{mg} / \mathrm{L}$, the residual turbidity of the supernatant, the water content of filter cake and the specific resistance of sludge decreased gradually with the increase of the dosage. When the dosage was greater than $40 \mathrm{mg} / \mathrm{L}$, the three indexes began to rise gradually, and the COD removal rate first increased to the highest value, then decreased slowly. In the process of flocculation, the positively charged P (AM-MAPTAC) flocculants can effectively compressed the electric double layer structure of sludge and further destroy its water film effect. In addition, the flocculants can also neutralize the negative charge on the surface of the sludge particles, so that the sludge particles can destabilize, gather and settle[19, 20]. Accordingly, the supernatant turbidity, moisture content of filter cake and the specific resistance of sludge decreased. Low CPAM dosage is not enough to neutralize the negative charge on the surface of sludge particles, and excess CPAM will stabilize the stabilized sludge particles, and thus not conducive to sludge flocculation. Therefore, the appropriate dosage of P(AM-MAPTAC) was deem to $40 \mathrm{mg} / \mathrm{L}$.
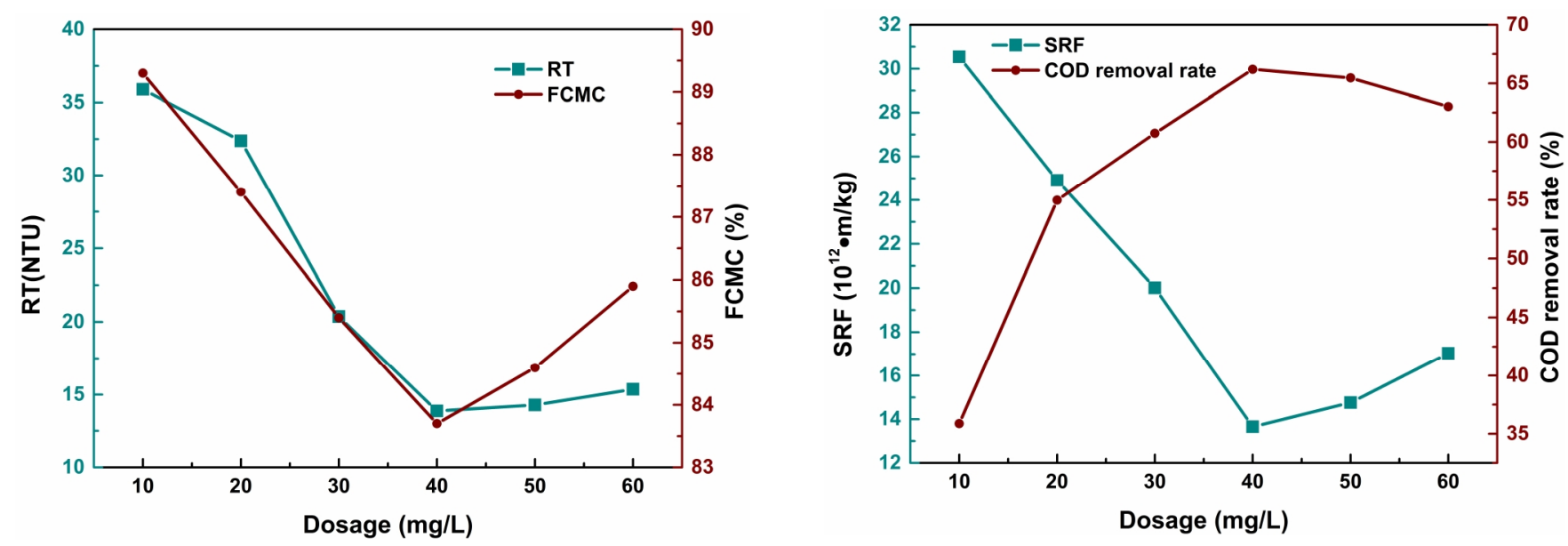

Figure 2. Effect of dosage on dewatering performance

\section{Effect of pH on dewatering performance}

The effect of pH on RT, FCMC, SRF and COD are shown in Fig. 3. RT, FCMC, SRF and COD removal rate were influenced by $\mathrm{pH}$ significantly. With the increase of $\mathrm{pH}$, the former three indexes first declined sharply to the minimum $(\mathrm{pH}=10.0)$ and then followed with a little bit back up. The optimal treatment effect achieved at $\mathrm{pH}=10.0$. The results showed that the alkaline condition was more beneficial to the flocculation of P (AM-MAPTAC) in the process of dyeing sludge dewatering. With the increasing of $\mathrm{pH}$ value, the existence of a large number of $\mathrm{OH}^{-}$will increase the number of negative surface charge of the sludge particles, and the P (AM-MAPTAC) flocculants can absorb negatively charged colloidal particles because of its cationic property, thus reducing the repulsive effect between the colloidal particles of sludge and making it easier to separate from the water[17, 21]. Therefore, the supernatant turbidity, moisture content of filter cake, sludge specific resistance is reduced, the removal rate of COD increased. It can be concluded that the optimum $\mathrm{pH}$ value should be controlled at about 10.0, when P (AM-MAPTAC) is used to adjust dyeing sludge. 

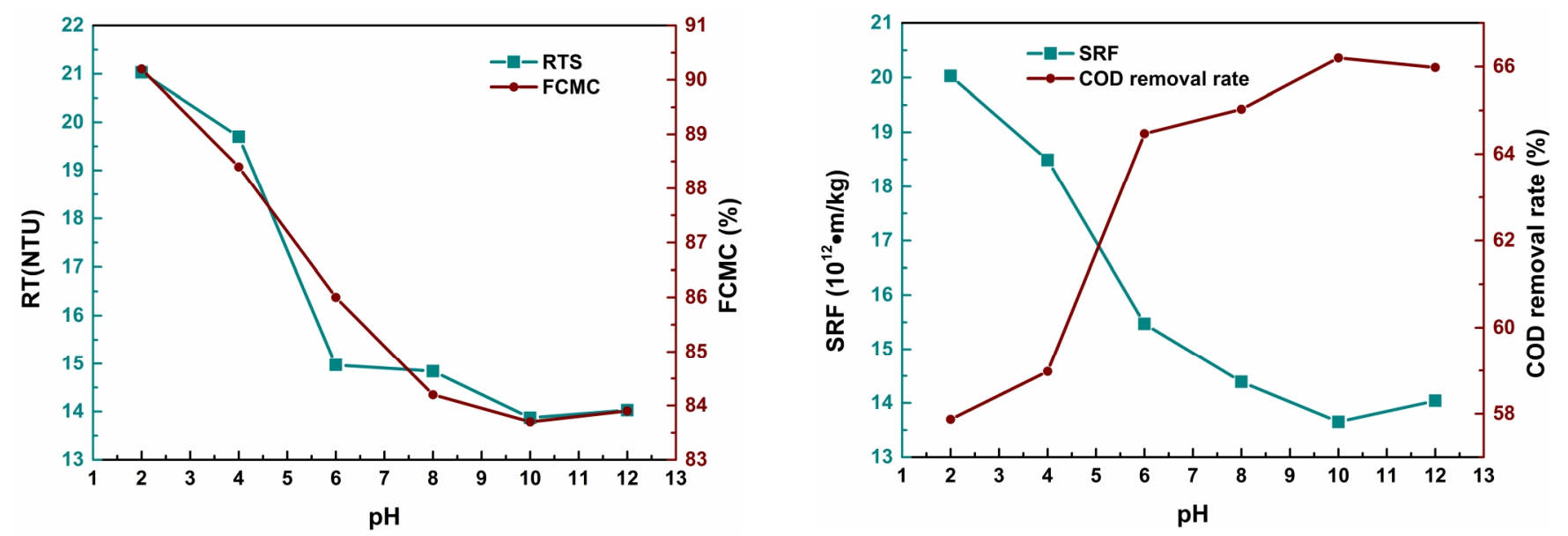

Figure 3. Effect of dosage on dewatering performance

\section{Effect comparison between $P$ (AM-MAPTAC) and commercial P(AM-DAC)}

The effect comparison between $\mathrm{P}$ (AM-MAPTAC) and commercial $\mathrm{P}(\mathrm{AM}-\mathrm{DAC})$ are shown in Fig. 4. After conducted by $\mathrm{P}$ (AM-MAPTAC) and $\mathrm{P}(\mathrm{AM}-\mathrm{DAC})$, with the rise of $\mathrm{pH}$, the residual turbidity and water content of filter cake decreased to the minimum and then increased slightly, and the minimum value all appeared at $\mathrm{pH}$ of about 10.0. Although the best treatment effect of $\mathrm{P}$ (AM-MAPTAC) flocculants was slightly lower than that of P(AM-DAC) flocculants, it showed better flocculation performance in most of the test range. Furthermore, with the $\mathrm{pH}$ value changing, $\mathrm{P}$ the flocculation performance of $\mathrm{P}(\mathrm{AM}-\mathrm{MAPTAC})$ was very stable, after processing the supernatant turbidity, cake moisture content remained at a low level. However, the dewatering effect of commercial $\mathrm{P}(\mathrm{AM}-\mathrm{DAC})$ on dyeing sludge was affected greatly by $\mathrm{pH}$ value, especially under acidic conditions, the flocculation effect of commercially $\mathrm{P}(\mathrm{AM}-\mathrm{DAC})$ were greatly deteriorated. Therefore, P (AM-MAPTAC) showed good acid and alkali resistance, and had better application value.
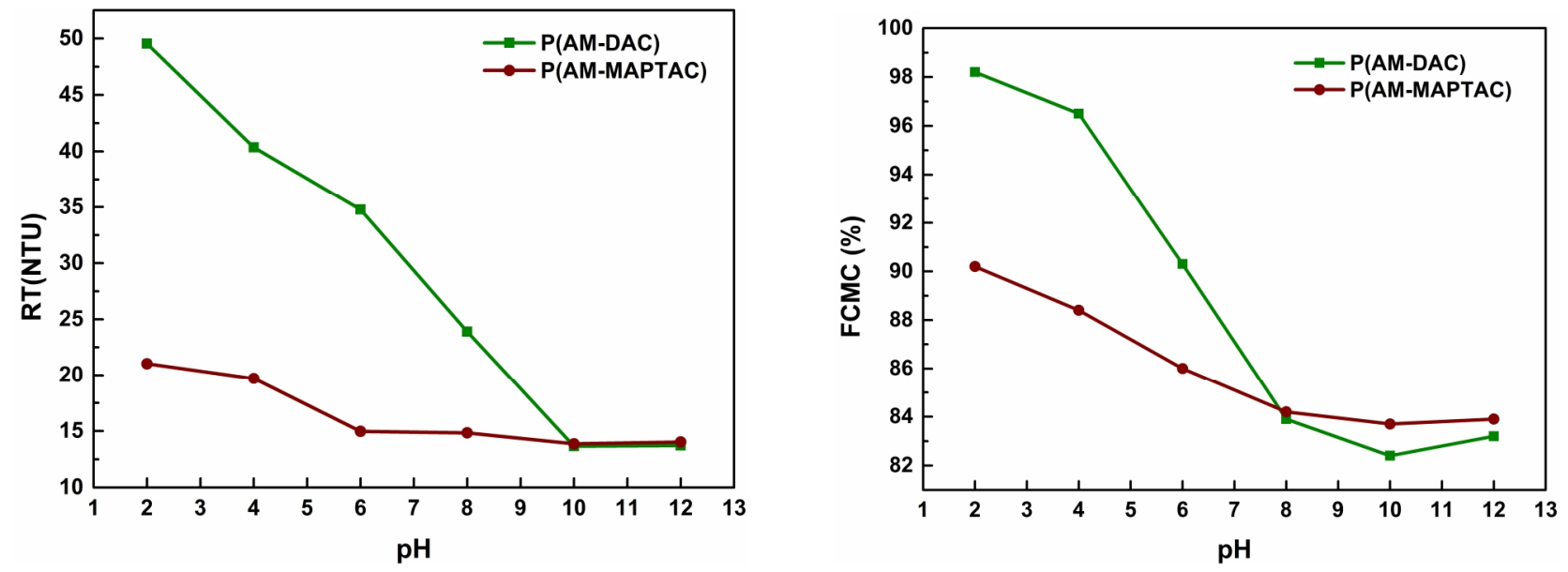

Figure 4. Effect comparison between P (AM-MAPTAC) and commercial P(AM-DAC)

\section{Conclusion}

In this work, AM and MAPTAC were successfully polymerized into P (AM-MAPTAC) through ultrasonic initiator system. The introduction of ultrasonic technology which featured initiated polymerization, stirring, dispersion and mixing functions, greatly shortened the copolymerization time. FTIR spectra of P(AM-MAPTAC) showed the existence of active groups of AM and MAPTAC and further confirmed the successful synthesis. TG/DSC analysis indicated that P (AM-MAPTAC) was quite stable under the conventional use and storage temperature of the flocculants, and the removal of 
major functional groups began at temperatures above $196.8^{\circ} \mathrm{C}$. P (AM-MAPTAC) showed excellent flocculation performance in dyeing sludge dewatering, and the following conclusions were obtained. 1) The appropriate $\mathrm{P}(\mathrm{AM}-\mathrm{MAPTAC})$ dosage for the flocculation of experimental dyeing sludge was 40 $\mathrm{mg} / \mathrm{L}$ and $14.80 \mathrm{NTU}$ of RT, $83.72 \%$ of FCMC, $66.8 \%$ of COD removal rate had been achieved, 2) The optimum $\mathrm{pH}$ value should be controlled at about 10.0, when P (AM-MAPTAC) is used to adjust dyeing sludge. 3) P (AM-MAPTAC) showed good acid and alkali resistance, and had better application value.

\section{Acknowledgements}

This work was financially supported by the National Natural Science Foundation of China (Project No. 21677020); the Education Committee of Sichuan Province (grant numbers 17ZB0299, 17ZB0313, 17ZB0312); the Sichuan University of Science and Technology level talent introduction project (grant numbers 2014RC32, 2016RCL20, 2016RCL06)

\section{References}

[1] Z. Huang, Y. Li, W. Chen, J. Shi, N. Zhang, X. Wang, Z. Li, L. Gao, Y. Zhang: Mater. Chem. Phys. Vol. 202 (2017), p. 266.

[2] H. Wu, Z. Liu, A. Li, H. Yang: Chemosphere Vol. 174 (2017), p. 200.

[3] H. Luo, X.-a. Ning, X. Liang, Y. Feng, J. Liu: Bioresource Technol. Vol. 139 (2013), p. 330.

[4] X.-a. Ning, W. Wen, Y. Zhang, R. Li, J. Sun, Y. Wang, Z. Yang, J. Liu: J. Environ.Manage. Vol.161 (2015), p. 181.

[5] Y. Liao, H. Zheng, L. Qian, Y. Sun, L. Dai, W. Xue: Indus. Eng. Chem. Res. Vol. 53 (2014), p. 11193.

[6] H. Zheng, Y. Sun, J. Guo, F. Li, W. Fan, Y. Liao, Q. Guan: Indus. Eng. Chem. Res. Vol. 53 (2014), p. 2572.

[7] L. Feng, H. Zheng, Y. Wang, S. Zhang, B. Xu: RSC Advan. Vol. 7 (2017), p. 23444.

[8] C. Zhao, H. Zheng, L. Feng, Y. Wang, Y. Liu, B. Liu, Z.B. Djibrine: Materials Vol. 10 (2017), 382.

[9] J. Wang, C. Chen, Q. Gao, T. Li, F. Zhu: Procedia Environ. Sci. Vol. 16 (2012), p. 409.

[10] J. Yang, S. Chen, H. Li: Int. Biodeter. \& Biodegr. https://doi.org/10.1016/j.ibiod.2016.10.027.

[11] X. Li, H. Zheng, B. Gao, Y. Sun, B. Liu, C. Zhao: Chemosphere Vol. 167 (2017), p. 71.

[12] X. Li, H. Zheng, B. Gao, C. Zhao, Y. Sun: Sep. Purif. Technol. Vol. 187 (2017), p. 244.

[13] H. Zheng, Y. Sun, C. Zhu, J. Guo, C. Zhao, Y. Liao, Q. Guan: Chem. Eng. J. Vol. 234 (2013), p. 318.

[14] J. Ma, K. Fu, J. Shi, Y. Sun, X. Zhang, L. Ding: Carbohyd. Polym. Vol. 151 (2016), p. 565.

[15] X. Li, H. Zheng, B. Gao, Y. Sun, X. Tang, B. Xu: RSC Adv. Vol. 7 (2017), p. 208.

[16] Z. Zhang, H. Zheng, F. Huang, X. Li, S. He, C. Zhao: Indus. Eng. Chem. Res. Vol. 55 (2016), p. 9819.

[17] W. Chen, H. Zheng, Q. Guan, H. Teng, C. Zhao, C. Zhao: Indus. Eng. Chem. Res. Vol. 55 (2016), p. 2892.

[18] Y. Liao, H. Zheng, Q. Li, Y. Sun, D. Li, W. Xue: Indus. Eng. Chem. Res. Vol. 53 (2014), p. 11193.

[19] J. Zhu, H. Zheng, Z. Jiang, Z. Zhang, L. Liu, Y. Sun: Desalin. Water Treat. Vol. 51 (2012), p. 2791.

[20] H. Zheng, Y. Sun, C. Zhu, J. Guo, C. Zhao, Y. Liao, Q. Guan: Chem. Eng. J. Vol. 234 (2013), p. 318.

[21] J. Ma, J. Shi, H. Ding, G. Zhu, K. Fu, X. Fu: Chem. Eng. J. Vol. 312 (2017), p. 20. 